\title{
MA-XRF for Historical Paintings: State of the Art and Perspective
}

\author{
Matthias Alfeld
}

TU Delft, Delft, Zuid-Holland, Netherlands

X-Ray Fluorescence imaging allows acquiring elemental distribution images by scanning the surface of a sample with a focused beam. While most popular for microscopic samples, dedicated instruments allow also the investigation of large objects by macroscopic XRF imaging (MA-XRF).

The non-destructive nature of MA-XRF investigations is well suited for the investigation of cultural heritage objects, such as historical paintings. The penetrative nature of X-rays allowed studying paint layers not only on the surface of paintings, but also below the surface. Early experiments on this saw this more as an oddity ([1] and references therein). The first experiment to gaining recognition was an experiment at the German Synchrotron DESY in Hamburg in that an discarded and overpainted study of a female peasant was found under the surface of an impressionistic flower still life by Vincent van Gogh[2].

First experiments were limited to synchrotron sources but were more dependent on their infrastructure than on the characteristics of the radiation. After all, paint layers are heavy metal salts embedded in a matrix of polymerized oil of up to several millimetre thickness, so that the radiation emitted by X-ray tubes is sufficient for scanning them. Further, did the limited amount of beam time and the effort in transporting an artwork to a synchrotron severely limited the number of paintings that could be investigated, albeit a number of studies was published [3-6]. A field in that synchrotron sources still excel is high lateral resolution MA-XRF due to the straightforward installation of advanced optics [7, 8].

Based on the experiences at synchrotron sources, mobile MA-XRF instruments using X-ray tubes were developed at the University of Antwerp [5, 9]. A number of instruments were developed at other institutes [10-12] and also commercial instruments were developed in the form of the Bruker M6 Jetstream [13] and the XGlab Crono [14]. These instruments allow scanning surfaces of approx. $50 \times 50 \mathrm{~cm}^{2}$ in a few hours. The lateral resolution can vary between a few tens to a few hundreds of micrometres, depending on the optics used and time available for the investigation. These instruments have allowed establishing MA-XRF with larger museums around the world having these instruments in their conservation workshops. It was shown to yield information similar to the established Neutron Activation Auto radiography with a much lower effort [15]. Also on site measurements in Etruscan and Egyptian tombs by MA-XRF have been reported $[16,17]$.

Today mature MA-XRF instruments exist that allow for a straightforward, reliable and rapid acquisition of data. This volume of data is, however, a problem. It is not reasonable to try to interpret hyperspectral data sets with hundreds of thousands of pixels and thousands of channels manually. Luckily, it is long established how to extract separate overlapping elemental signals in XRF data (see [18] and references there in).

However, even if the MA-XRF data is reduced to 10 different elemental distribution images it is often not directly legible by humans as it contains a significant degree of redundancies and hidden correlations. These characteristics prevent a straightforward interpretation of data, especially if large data sets of several paintings are to be interpreted. 
As opposed to other samples paintings consist of a three dimensional superposition of different paint layers semi-transparent to X-rays. This results in artefacts due to self-absorption. These artefacts can be corrected by dedicated data treatment $[5,19]$. This further limits the usage of simple hard clustering approaches in data processing. Likewise, PCA allows for a reduction of the data set but does not necessarily yield a more legible representation. However, a number of approaches were taken.

Non-Negative Matrix Factorization (NMF) allows to factorise a data set into coefficients and basis vectors (similar to scores and loads in PCA) with a non-negative constraint. It has been successfully applied for the treatment of raw spectral data and elemental distribution images yielded by deconvolution [20-23].

Instead of determining basis vectors (loads in PCA) iteratively, once can search in a data set for archetypes, extreme data points, and express the entirety of the data set as a linear combination of these archetypes. One approach to this is Simplex Volume Maximisation (SiVM), which was successfully applied to a painting by Hans Memling [24].

Recently, the complementary nature of Reflectance Imaging Spectroscopy (RIS) in the visible and near IR range and MA-XRF has been more and more exploited [25, 26]. A direct fusion of RIS and MA-XRF data and the joint evaluation via t-Stochastic Neighbour Embedding (t-SNE) was recently described [17].

This presentation will summarize the state of the art in MA-XRF for historical paintings and the chemometric approaches for handling and clustering the data. It will further discuss the perspective for further developments in the field of art-analysis, especially related to MA-XRF.

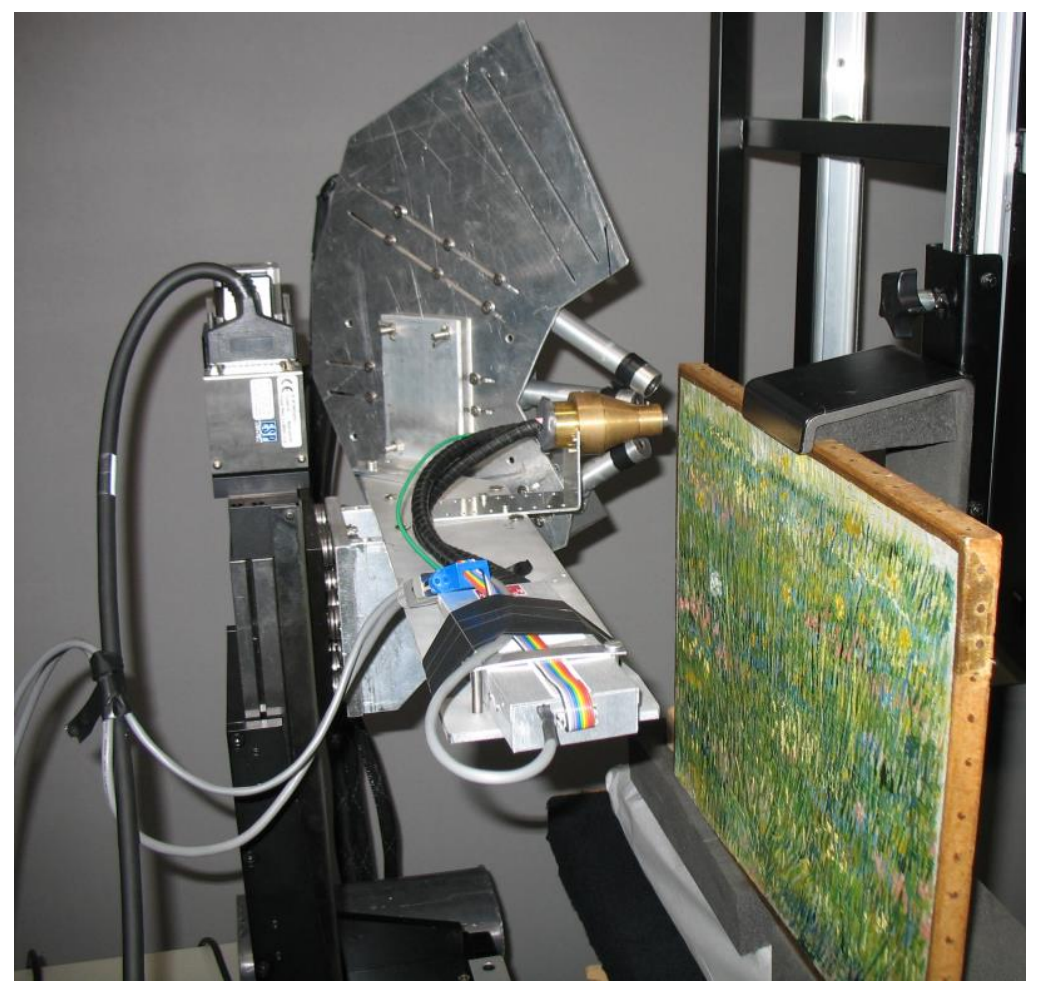

Figure 1. In-house build MA-XRF scanner of the University of Antwerp while investigating Vincent van Gogh's Patch of Grass. 

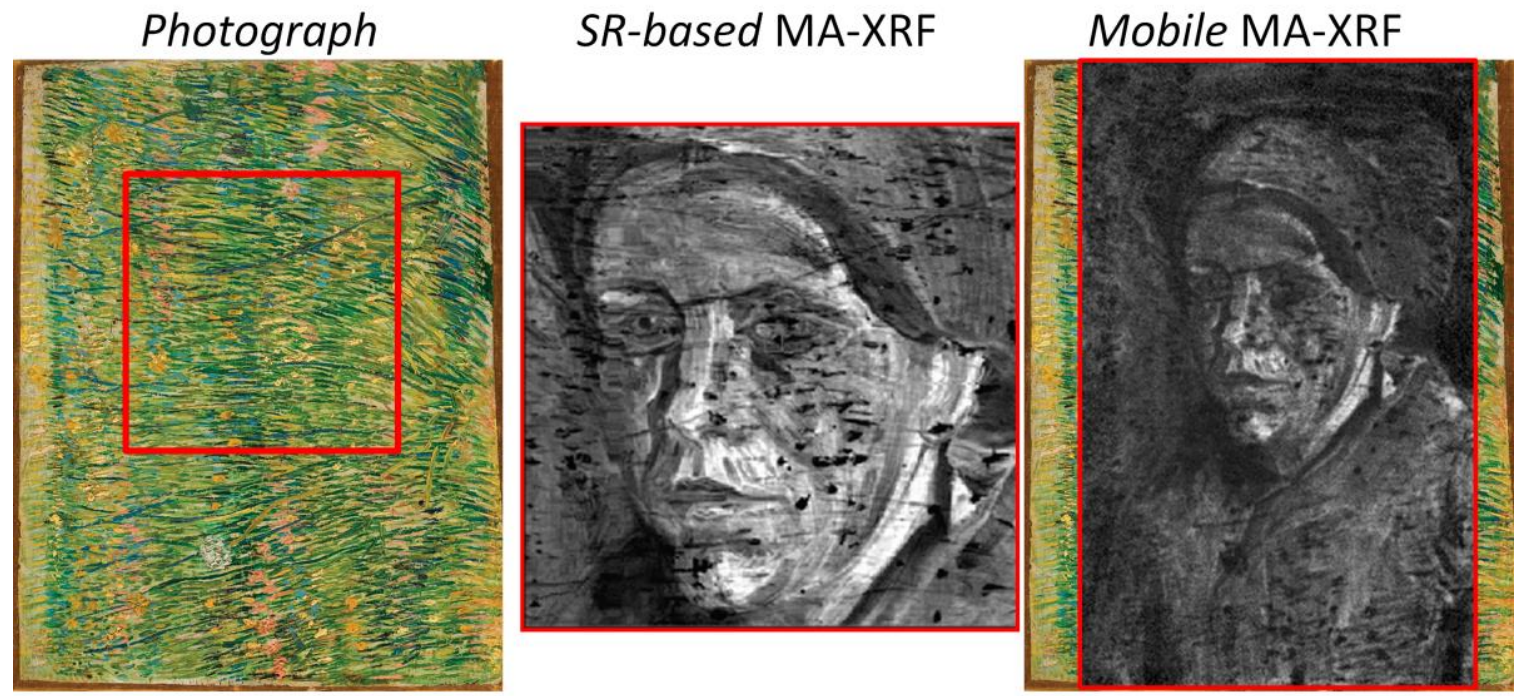

Figure 2. Sb distribution images acquired on Vincent van Gogh's Patch of Grass indicating the subsurface distribution of Naples Yellow (Pb2Sb2O7). Left: Photograph, rotated by 90 degrees, red shape indicates Synchrotron experiments [2]. Centre: Results obtained at DESY synchrotron. Right: Results obtained by mobile MA-XRF instrument [9].

References

1. M. Alfeld and J. A. C. Broekaert, Spectrochim. Acta B 88, 211 (2013).

2. J. Dik, K. Janssens, G. Van der Snickt, L. van der Loeff, K. Rickers, and M. Cotte, Anal. Chem. 80, 6436 (2008).

3. M. Alfeld, et al., Appl. Phys. A 111, 157 (2013).

4. M. Alfeld, et al., J. Anal. At. Spectrom. 28, 40 (2013).

5. M. Alfeld, et al., Appl. Phys. A 111, 165 (2013).

6. M. Alfeld, et al., Zeitschrift für Kunsttechnologie und Konserv. 25, 157 (2011).

7. D. Thurrowgood, et al., Sci. Rep. 6, 29594 (2016).

8. D. L. Howard, et al., Anal. Chem. 84, 3278 (2012).

9. M. Alfeld, et al., J. Anal. At. Spectrom. 26, 899 (2011).

10. F. P. Romano, et al., J. Anal. At. Spectrom. 32, 773 (2017).

11. E. Ravaud, et al., Appl. Phys. A 122, 17 (2016).

12. F.-P. Hocquet, et al., Anal. Bioanal. Chem. 399, 3109 (2011).

13. M. Alfeld, et al., J. Anal. At. Spectrom. 28, 760 (2013).

14. R. Alberti, et al., X-Ray Spectrom. 46, 297 (2017).

15. M. Alfeld, et al., Appl. Phys. A 119, 795 (2015).

16. M. Alfeld, et al., X-Ray Spectrom. 48, 262 (2019).

17. M. Alfeld, et al., Comptes Rendus Phys. 19, 625 (2018).

18. M. Alfeld and K. Janssens, J. Anal. At. Spectrom. 30 (2015).

19. P. M. Wróbel, et al., Anal. Chem. 88, 1661 (2016).

20. A. Martins, et al., Herit. Sci. 4, 33 (2016).

21. A. Martins, et al., Herit. Sci. 4, 22 (2016).

22. M. Alfeld, et al., J. Phys. Conf. Ser. 499, 012013 (2014).

23. H. C. Santos, et al., Microchem. J. 124, 241 (2016).

24. M. Alfeld, et al., Microchem. J. 132, 179 (2017). 
25. K. A. Dooley, et al., Herit. Sci. 6, 46 (2018).

26. G. Van der Snickt, et al., Appl. Spectrosc. 70,57 (2016). 